\title{
Revestimentos cerâmicos utilizados como barreira térmica
}

\section{(Ceramic coatings used as thermal barrier)}

\author{
J. P. Oliveira, J. F. Duarte \\ CENIMAT/I3N, Departamento de Ciência dos Materiais, Faculdade de Ciências e Tecnologia, FCT, \\ Universidade Nova de Lisboa, 2829-516 Caparica, Portugal \\ jp.oliveira@campus.fct.unl.pt,jf.duarte@campus.fct.unl.pt
}

\begin{abstract}
Resumo
Neste trabalho procurou-se fazer uma introdução aos revestimentos de barreira térmica cerâmicos, em especial à utilização deste tipo de materiais na camada top coat, apresentando as suas propriedades fundamentais. Posteriormente apresentam-se os novos materiais que podem vir a substituir a zircónia parcialmente estabilizada com ítria, sendo este o material mais utilizado para desempenhar esta função. Neste trabalho, também se faz uma descrição sumária dos métodos de processamento de cada material que foram abordados neste trabalho. E por fim apresenta-se uma tabela com algumas das propriedades termo-físicas mais importantes para os materiais analisados
\end{abstract}

Palavras-chave: revestimentos de barreira térmica, TBC's, PSZ.

\begin{abstract}
In this work we started by doing a introduction to the ceramic thermal barrier coatings, especially in the utilization of this kind of material as top coat by presenting its fundamental properties. After we present new materials that may replace zirconia partially stabilized with yttria (PSZ), being this last material the most used as top coat. In this work, we also make a brief description of the processing methods for each material analyzed in this article. Last we present a table when some of the most important thermalphysical properties of the materials studied.
\end{abstract}

Keywords: thermal barrier coatings, TBCS, PSZ.

\section{INTRODUÇÃO}

Durante as últimas décadas têm sido feitos esforços no sentido de desenvolver e produzir revestimentos cerâmicos utilizados como barreira térmica, TBCs do inglês "thermal barrier coatings", para serem utilizados em turbinas, por exemplo, uma vez que os materiais que eram utilizados para esse efeito atingiram o máximo das suas capacidades em termos de temperatura que são capazes de suportar. Os TBCs são sistemas que utilizam materiais dito avançados e que são normalmente aplicados em superfícies metálicas em zonas onde as temperaturas envolvidas sejam muito elevadas. Estes revestimentos servem como isoladores de componentes que estão sujeitos a elevadas temperaturas durante períodos significativos, protegendo-os assim da exposição térmica que pode provocar a sua degradação. Os TBCs são utilizados em peças de transição, linhas de combustão e em zonas sujeitas a elevadas temperatura, como em turbinas, de modo a permitir a entrada de ainda maiores temperaturas para que haja um aumento da eficiência ou uma diminuição dos requisitos dos sistemas de refrigeração [1]. Geralmente os revestimentos cerâmicos são utilizados em conjunto com revestimentos metálicos e devido à diferença de condutividades térmicas entre os dois tipos de materiais é possível reduzir a temperatura dos substratos metálicos levando a um consequente aumento do seu tempo de vida, bem como, a possibilidade de aumentar a temperatura de funcionamento nas turbinas, por exemplo.

Geralmente os sistemas de revestimento de barreira térmica consistem em duas camadas, uma chamada de bond coat, ou revestimento de ligação, e uma outra, isolante, chamada de top coat, feita de material cerâmico. Será sobre os materiais possíveis de serem utilizados nesta camada que este trabalho versará. A camada bond coat é normalmente um metal e têm duas funções essenciais: melhorar a ligação entre o substrato e o top coat e proteger o substrato da corrosão e oxidação [2]. Geralmente tem-se o crescimento de um óxido entre o cerâmico e o metal devido à elevada temperatura a que estas camadas estão sujeitas. Nas Figs. 1 [3] e 2 [4] apresentam-se uma vista em corte de um sistema TBC genérico e um esquema de TBC para turbinas, respectivamente.

As propriedades fundamentais que um material cerâmico deve possuir, para ser utilizado como top coat em sistemas TBC são: baixa condutividade térmica, coeficiente de expansão semelhante ao do material utilizado como bond coat de modo a evitar a existência de tensões de origem térmica, ponto de fusão elevado e boas propriedades mecânicas a elevadas temperaturas.

Os primeiros revestimentos cerâmicos foram desenvolvidos pela National Advisory Commitee for Aeronautics. Um revestimento à base de zircónia $\left(\mathrm{ZrO}_{2}\right)$ 


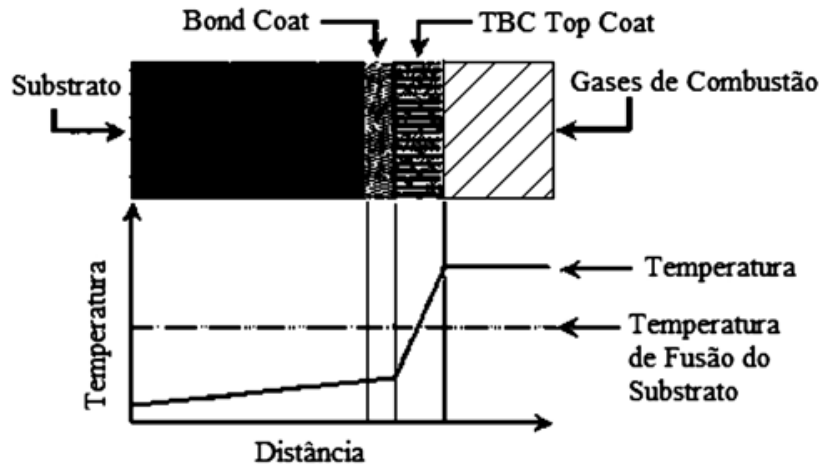

Figura 1: Vista em corte de um esquema geral de um sistema TBC, onde identifica as camadas bond coat e top coat, bem como o substrato adjacente ao bond coat.

[Figure 1: Sectional view of the general scheme of one TBC system, where is visible the bond coat and top coat layers are as well the adjacent subtract to the bond coat layer.]

estabilizada com cálcia $(\mathrm{CaO})$ num bocal de exaustão de um avião na década de 60 foi o primeiro TBC utilizado em voos tripulados. [5] Foi no entanto, na década de 80 que os TBCs tiveram uma melhoria significativa devido à utilização de zircónia parcialmente estabilizada com ítria $\left(\mathrm{Y}_{2} \mathrm{O}_{3}\right)$, PSZ. Este cerâmico demonstrou ter propriedades excepcionais, como elevada tenacidade à fractura, para ser utilizado como top coat e têm sido o material de referência para este tipo de aplicações nos últimos 30 anos.

A utilização de novos materiais cerâmicos em TBCs passa necessariamente por terem propriedades superiores às da PSZ. No caso da PSZ as suas propriedades relevantes são limitadas a uma temperatura de $1200{ }^{\circ} \mathrm{C}$ [6], pelo que a sua substituição enquanto material para top coat terá de ser feita através da utilização de materiais com propriedades suficientemente atractivas acima dessas temperaturas.

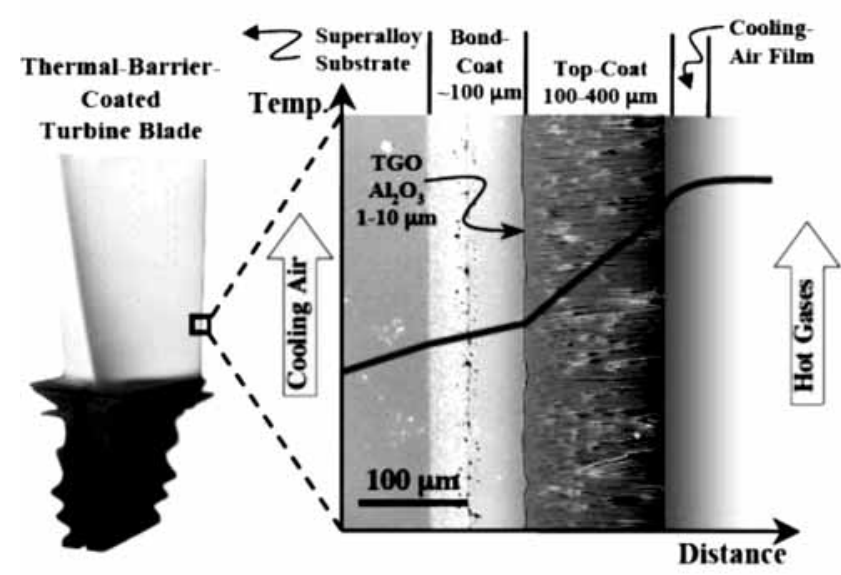

Figura 2: Esquema de um TBC para turbinas. A linha representa o aumento da temperatura em função da distância ao substrato metálico.

[Figure 2: Scheme of one TBC for turbines. The line represents the increase of the temperature as a function to the distance to the metallic subtract.]
Além das já mencionadas turbinas diversas zonas dos aviões estão sujeitas a tensões mecânicas, térmicas e químicas, pelo que é necessário assegurar que os materiais utilizados possuam uma elevada resistência a este tipo de solicitações. É aqui que entram os materiais cerâmicos, uma vez que comparativamente com os metais, possuem uma maior resistência à oxidação, corrosão e desgaste, sendo igualmente melhores isoladores térmicos.

\section{MÉTODOS DE PROCESSAMENTO DE MATERIAIS CERÂMICOS PARA TBC's}

Relativamente aos métodos de fabrico dos TBCs existem dois métodos com provas dadas. O primeiro é a deposição física de vapores utilizando feixe de electrões ${ }^{1}$, EB-PVD, e o segundo método é a pulverização por plasma à pressão atmosférica $^{2}$, APS. Os revestimentos depositados através de EB-PVD apresentam microestruturas do tipo colunar e são normalmente utilizados em pás submetidas a esforços termo-mecânicos elevados de motores para aviões. A técnica de APS é mais utilizada do que a EB-PVD devido a ser um processo mais económico e mais fácil de utilizar. Normalmente componentes estáticos, como as turbinas, cilindros de combustão, e lâminas das hélices são revestidos através do processo de APS. A promoção da eficiência nas turbinas é procurada através do desenvolvimento de novas tecnologias de arrefecimento e de combustão combinadas com a admissão de maiores temperaturas nestes componentes, no sentido de aumentar a sua eficiência. Estes factores levam a que o material mais comummente utilizado até agora, a PSZ, esteja a atingir os seus limites especialmente devido às transformações de fase que podem ocorrer em virtude do aumento da temperatura.

A PSZ formada através de EB-PVD ou APS consiste numa fase T' metaestável. Quando ocorrem exposições prolongadas a elevadas temperaturas, dá-se a decomposição em fases com elevado teor de ítria, fases cúbicas, e fases com baixo teor de ítria, fases monoclínicas. A fase monoclínica quando em arrefecimento tem associada um aumento do seu volume o que pode provocar falhas do tipo catastrófico nos TBCs, quando em funcionamento. Tal como foi anteriormente referido o limite em termos de temperatura para a utilização de PSZ em TBCs é de $1200^{\circ} \mathrm{C}$. Juntamente com a capacidade limitada para se ter uma fase estável existe ainda o problema de ocorrer sinterização do material em virtude das elevadas temperaturas, o que provoca uma menor capacidade de deformação dos revestimentos levando a que a sua falha em serviço possa ocorrer precocemente [7]. Na Fig. 3 [8] apresenta-se o diagrama de fases das PSZ.

\footnotetext{
${ }^{1}$ Nesta técnica o feixe de electrões no alvo leva a que os átomos do alvo sofrem uma transformação para o estado gasoso. Em seguida esses átomos vão precipitar na forma sólida, revestindo toda câmara onde ocorre o processo, bem como o objecto que se pretende efectivamente revestir.

${ }^{2}$ Nesta técnica faz-se a pulverização do material que se pretende depositar. O material a depositar está na forma fundida para que a pulverização seja facilitada.
} 


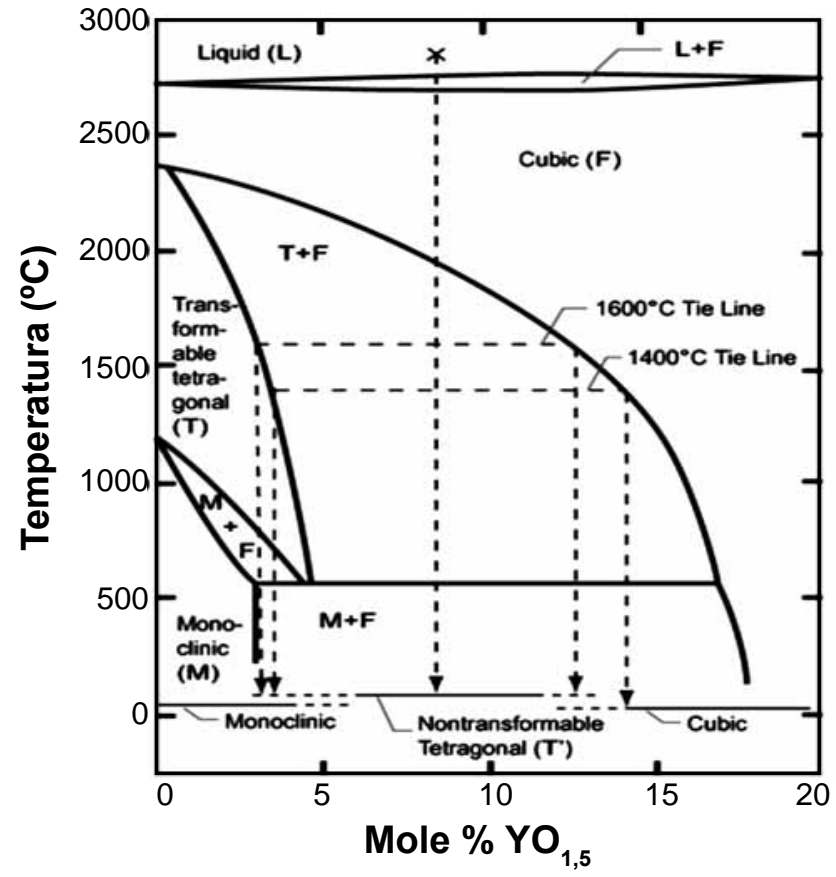

Figura 3: Diagrama de fases da zircónia parcialmente estabilizada com ítria.

[Figure 3: Phase diagram for the zirconia partially stabilized with itria.]

\section{MATERIAIS PARA UTILIZAÇÃO EM TBC's}

\section{Pirocloros}

Este tipo de materiais com uma estrutura típica do mineral pirocloro, do tipo $\mathrm{A}_{2} \mathrm{~B}_{2} \mathrm{O}_{7}$ ou $\mathrm{A}_{2} \mathrm{~B}_{2} \mathrm{O}_{6}$, onde $\mathrm{A}$ e $\mathrm{B}$ são, em geral, terras-raras ou metais de transição, oferece propriedades bastante interessantes para aplicações sujeitas a temperaturas superiores a $1300^{\circ} \mathrm{C}$. Especificamente a baixa condutividade térmica de bastantes zirconatos, provoca um grande interesse nesta classe de materiais. Outra propriedade relevante é a sua elevada estabilidade térmica, e que está provavelmente relacionada com as posições fixas dos catiões no cristal. Os pirocloros mais estudados são os zirconatos à base de terras-raras, do tipo $\mathrm{Ln}_{2} \mathrm{Zr}_{2} \mathrm{O}_{7}$, onde $\mathrm{Ln}$ pode ser La, $\mathrm{Gd}, \mathrm{Sm}, \mathrm{Nd}$, Eu ou $\mathrm{Yb}$. Outros materiais são também alvo de estudo intensivo, em especial compostos baseados em Ce como $\mathrm{La}_{2} \mathrm{Ce}_{2} \mathrm{O}_{7}$ ou $\mathrm{La}_{2}\left(\mathrm{Zr}_{0.7} \mathrm{Ce}_{0.3}\right)_{2} \mathrm{O}_{7}$. Na verdade os óxidos baseados em Ce mostram muitas vezes uma estrutura do tipo da fluorite com defeitos ${ }^{3}$. É esta estrutura que permite uma troca mais fácil entre catiões, o que pode ajudar a explicar a elevadas taxas de sinterização para este tipo de materiais. A dopagem destes materiais com elementos como Y leva a que haja uma maior resistência à sinterização. Dentro dos pirocloros o composto mais promissor é o $\mathrm{La}_{2} \mathrm{Zr}_{2} \mathrm{O}_{7}$ (LZ) o que apresenta características mais interessantes para aplicação como TBC. Quando comparado com a PSZ este

${ }^{3}$ A estrutura da fluorite (CaF2) com defeitos serve para mostrar que a dopagem efetuada nos materiais mencionados neste capítulo não mantém a estrutura inicial, provocando sim alguns defeitos. composto apresenta estabilidade térmica superior a $2000^{\circ} \mathrm{C}$, uma condutividade térmica inferior a $2 \mathrm{~W} / \mathrm{mK}$ e uma baixa tendência para sinterizar. No entanto o seu baixo coeficiente de expansão (cerca de $9,1 \times 10^{-6} \mathrm{~K}^{-1}$ vs $11,5 \times 10^{-6} \mathrm{~K}^{-1}$, no caso da PSZ) provoca maiores tensões térmicas devido a incompatibilidades na expansão térmica decorrentes da sobreposição com outros materiais que envolvem o TBC, que por possuírem maiores coeficientes de expansão térmica levam à existência de tensões internas nos materiais.

Dado que, em geral, tanto o substrato como o bond coat possuem maiores coeficientes de expansão térmica (na ordem dos $\left.15 \times 10^{-6} \mathrm{~K}^{-1}\right)$, podem ocorrer facilmente fissuras devido às tensões acumuladas pelo aumento da temperatura durante $o$ funcionamento da aplicação em questão. Este problema pode ser ultrapassado através de um sistema de duas camadas. A primeira camada irá conter PSZ e a camada superior contém um material à base de pirocloros, levando a um aumento do tempo de vida dos revestimentos em gradientes de temperatura cíclicos. Esta dupla camada providencia uma boa tenacidade, semelhante à do bond coat, e a utilização de pirocloros leva a uma maior estabilidade térmica e a uma menor ocorrência de sinterização. Esta solução pode ser utilizada para substituir a PSZ como componente único em TBCs, uma vez que os resultados evidenciam propriedades superiores a elevadas temperaturas [9]. A utilização deste tipo de sistemas pode levar à capacidade de aumentar mais de $100{ }^{\circ} \mathrm{C}$ a temperatura nas turbinas. Apesar das boas propriedades a elevada temperatura deste sistema de dupla camada já terem sido provadas é ainda necessário melhorar a sua performance para temperaturas mais reduzidas, uma vez que para temperaturas inferiores as propriedades obtidas não são tão interessantes como aquelas obtidas a altas temperaturas. Neste campo existem alguns resultados experimentais contraditórios, que necessitam de um maior estudo $[10,11]$.

\section{"Cluster TBC"}

Este tipo de materiais é um desenvolvimento bastante recente tendo como principal impulsor a NASA. Neste tipo de materiais faz-se a dopagem de zircónia com terrasraras. Estas adições levam à formação de agregados de dopantes, com sistemas complexos do tipo $\mathrm{ZrO}_{2}-\mathrm{Y}_{2} \mathrm{O}_{3}-$ $\mathrm{Nd}_{2} \mathrm{O}_{3}\left(\mathrm{Gd}_{2} \mathrm{O}_{3}, \mathrm{Sm}_{2} \mathrm{O}_{3}\right)-\mathrm{Yb}_{2} \mathrm{O}_{3}\left(\mathrm{Sc}_{2} \mathrm{O}_{3}\right)$. Este tipo de sistema é capaz de provocar uma diminuição significativa da condutividade térmica, entre 20 a $40 \%$.

A adição de terras-raras promove igualmente a estabilidade térmica dos revestimentos obtidos. Em termos de performance comparativamente com a PSZ, este tipo de materiais apresenta resultados semelhantes ou ligeiramente superiores. Além disso é possível utilizar estes materiais para sistemas que estejam sujeitos a temperaturas até $1650{ }^{\circ} \mathrm{C}$, o que é uma diferença em mais de $400{ }^{\circ} \mathrm{C}$ relativamente às temperaturas suportadas pela PSZ [12].

A utilização de grandes quantidades de dopante leva à promoção da uma fase cúbica estabilizada, no entanto, temse uma ligeira diminuição da tenacidade com consequência 
para o desempenho do material enquanto revestimento.

Tal como nos pirocloros a utilização de uma dupla camada é igualmente vantajosa, levando a um aumento do número de ciclos que o revestimento é capaz de suportar, podendo variar entre os 100 a 300 ciclos extra.

\section{Hexaaluminatos}

Os hexaaluminatos de lantanídeos com estrutura do tipo magnetoplumbítico (estrutura apresentada na Fig. 4 [13]) são actualmente utilizados em vários campos da ciência como na tecnologia laser, catálise e em aplicações magnéticas. [14] Devido à sua elevada temperatura de fusão, coeficiente de expansão térmico elevado, baixa condutividade térmica, resistência à sinterização durante bastante tempo e estabilidade estrutural até $1800^{\circ} \mathrm{C}$, este tipo de materiais tem sido visto como um potencial candidato para ser utilizado em TBCs.

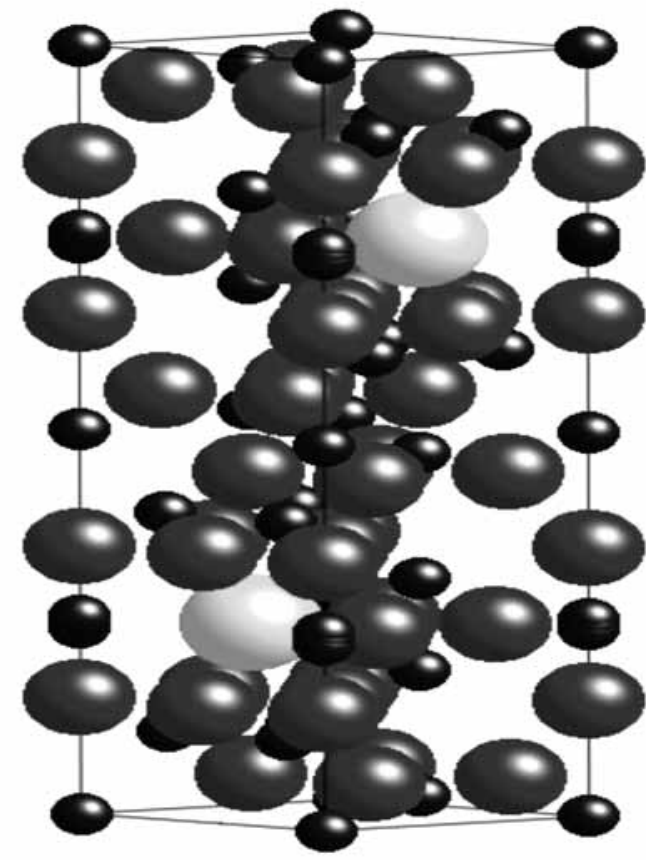

Figura 4: Estrutura do tipo magnetoplumbítico.

[Figure 4: Magneto-plumbite structure.]

A composição deste tipo de material é do tipo (La,Nd) $\mathrm{MAl}_{11} \mathrm{O}_{19}$ onde $\mathrm{M}$ pode ser $\mathrm{Mg}, \mathrm{Mn}, \mathrm{Sm}, \mathrm{Cr}$ ou $\mathrm{Zr}$. Entre as diferentes possibilidades existentes o composto $\mathrm{LaMgAl}_{11} \mathrm{O}_{19}$ é aquele que tem sido mais estudado, quer em termos das suas propriedades termo-físicas como também dos problemas decorrentes do seu processamento, feito, geralmente, recorrendo à técnica de APS [15]. O revestimento obtido através da técnica de APS é parcialmente amorfo devido ao arrefecimento extremamente rápido por parte do material que anteriormente estava no estado fundido. A aplicação de um tratamento térmico após a sua deposição possibilita uma recristalização do material, levando também a uma diminuição significativa do seu volume [16].

A diminuição de volume verificada é atribuída à cristalização parcial da zona amorfa do composto pode estar relacionada com a ocorrência de uma transformação de fases que ocorre por passos, até se chegar à fase estável. As fases secundárias que se formarão partem do sistema La-Al-Mg. Em particular podem ser observadas fases peroviskíticas ${ }^{4}$ como $\mathrm{LaAlO}_{3}$ a temperaturas inferiores a $1400{ }^{\circ} \mathrm{C}$, antes da transformação de fases do hexaaluminato estar concluída acima dos $1500{ }^{\circ} \mathrm{C}$ [17]. Na maior parte dos hexaaluminatos produzidos via APS tem-se o desenvolvimento de uma fragmentação na rede cristalina que leva a um aumento da tolerância de deformação dos revestimentos e consequentemente a uma maior resistência ao choque térmico o que é extremamente útil nos TBCs [18]. Em termos de desenvolvimentos mais recentes e com possível aplicação no futuro foi proposto a utilização de compósitos de alumina, $\mathrm{Al}_{2} \mathrm{O}_{3}$, e hexaaluminatos de $\mathrm{La}$ para a utilização como TBCs uma vez que estes compósitos possuem uma maior ductilidade e uma tenacidade à fractura superiores [19].

\section{Perovskites}

Este tipo de estruturas pode acomodar uma grande variedade de iões em solução sólida, incluindo iões com massas atómicas mais elevadas. A maior parte destes materiais, com fórmula química do tipo $\mathrm{ABO}_{3}$, é estável a elevadas temperaturas levando a que sejam vistos como candidatos para o desenvolvimento de aplicações utilizando TBCs. Na Fig. 5 [20] apresenta-se a estrutura típica da perovskite.

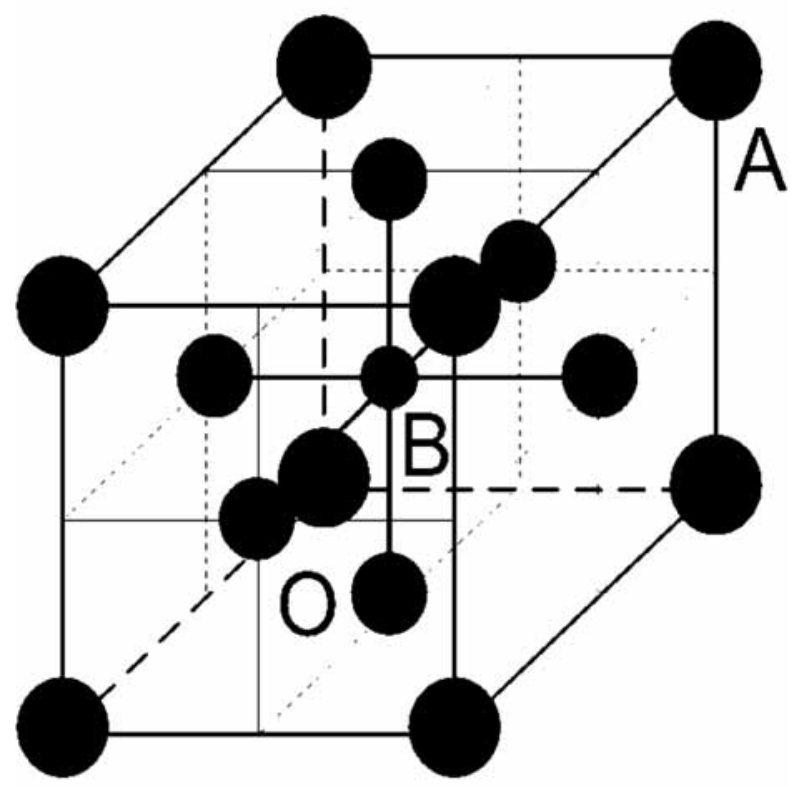

Figura 5: Estrutura típica da perovskite. [Figure 5: Typical structure of perovskite.]

${ }^{4}$ Este tipo de estruturas tem fórmula química do tipo $\mathrm{ABO3}$, onde $A$ e $B$ são catiões com diferentes raios atómicos. A sua estrutura é geralmente do tipo tetragonal. 


\section{Zirconatos}

Um dos primeiros candidatos para ser utilizados como TBC foi o $\mathrm{BaZrO}_{3}$. Apesar de possuir um ponto de fusão de $2600{ }^{\circ} \mathrm{C}$, possui baixa estabilidade térmica e química o que leva a que possam ocorrer falhas precoces quando sujeito a esforços mecânicos a temperaturas na ordem dos $120{ }^{\circ} \mathrm{C}$ [21].

A utilização de $\mathrm{SrZrO}_{3}$ pode ser viável uma vez que este material apresenta boas propriedades a temperaturas superiores a $1250{ }^{\circ} \mathrm{C}$, quer seja utilizado sozinho ou num sistema de dupla camada com PSZ. A uma temperatura de cerca de $730{ }^{\circ} \mathrm{C}$ o $\mathrm{SrZrO}_{3}$ sofre uma transformação de fase indesejável passando de uma estrutura ortorrômbica para uma estrutura pseudo-tetragonal, que pode provocar problemas devido à variação de volume que ocorre. Esta transformação pode, no entanto, ser suprimida através da dopagem com Gd ou Yb, que levam igualmente a uma melhoria das propriedades termo-físicas deste material a elevadas temperaturas [22].

$\mathrm{O}$ mais recente material a despertar atenções para poder ser utilizado para TBCs é o $\mathrm{CaZrO}_{3}$. Ainda que tenha um ponto de fusão inferior ao da PSZ tem uma condutividade térmica interessante, na ordem dos $2 \mathrm{~W} / \mathrm{mK}$.

\section{Formas complexas}

Além da elevada temperatura de fusão, uma característica interessante sobressai das perovisktites ditas complexas, que é o facto de se poder jogar com o efeito do catião B da estrutura da peroviskite, que leva a que as propriedades deste tipo de materiais possa ser manipulada consoante a escolha desse catião. Por exemplo, o $\mathrm{Ba}\left(\mathrm{Mg}_{1 / 3} \mathrm{Ta}_{2 / 3}\right) \mathrm{O}_{3}$ possui uma fase cúbica desordenada, podendo, no entanto, ser promovida uma fase ordenada trigonal através da utilização de uma temperatura superior a $1650{ }^{\circ} \mathrm{C}$ durante o processo de síntese deste material.

Geralmente, durante o processo APS os revestimentos obtidos apresentam uma estrutura desordenada, o que leva a que não se consiga promover a existência de propriedades homogéneas em todo o revestimento. Este problema pode ser ultrapassado através de um recozimento a temperaturas superiores a $1250{ }^{\circ} \mathrm{C}$, que leva a que o revestimento fique com uma estrutura ordenada.

Outro material que apresenta características interessantes é o $\mathrm{La}\left(\mathrm{Al}_{1 / 4} \mathrm{Mg}_{1 / 2} \mathrm{Ta}_{1 / 4}\right) \mathrm{O}_{3}$, uma vez que durante o seu processamento através de APS obtém-se novamente fragmentação da rede cristalina, o que é favorável para aplicações em TBCs devido ao facto de propiciar uma maior

Tabela I - Resumo de algumas propriedades termo-físicas dos materiais abordados neste trabalho. [Table I - Summary of some thermo-physical properties of the materials addressed in this work.]

\begin{tabular}{|c|c|c|c|c|c|}
\hline Material & $\begin{array}{l}\text { Temperatura } \\
\text { de fusão } \\
{\left[{ }^{\circ} \mathrm{C}\right]}\end{array}$ & $\begin{array}{c}\text { Coeficiente } \\
\text { de expansão } \\
\text { térmica } \\
\text { de } 30 \text { a } 1000{ }^{\circ} \mathrm{C} \\
{\left[10^{-6} \mathrm{~K}^{-1}\right]}\end{array}$ & $\begin{array}{c}\text { Condutividade } \\
\text { térmica a } \\
1000^{\circ} \mathrm{C} \\
{[\mathrm{W} / \mathrm{mK}]}\end{array}$ & $\begin{array}{c}\text { Módulo } \\
\text { de Young } \\
\text { [GPa] }\end{array}$ & $\begin{array}{c}\text { Tenacidade à } \\
\text { fractura } \\
{\left[\mathrm{MPa} \mathrm{m}^{1 / 2}\right]}\end{array}$ \\
\hline PSZ & 2680 & 11,5 & 2,12 & 210 & $1-2$ \\
\hline \multicolumn{6}{|l|}{ Pirocloros } \\
\hline $\mathrm{La}_{2} \mathrm{Zr}_{2} \mathrm{O}_{7}$ & 2300 & 9,1 & 1,56 & 175 & \\
\hline $\mathrm{Gd}_{2} \mathrm{Zr}_{2} \mathrm{O}_{7}$ & 2200 & 10,4 & 1,6 & & \\
\hline Cluster TBC & & $11,5-13,5$ & $1,7-2$ & & \\
\hline \multicolumn{6}{|l|}{ Hexaaluminatos } \\
\hline $\mathrm{LaMgAl}_{11} \mathrm{O}_{19}$ & & 9,5 & 2,7 & 130 & \\
\hline $\mathrm{GdMgAl}_{11} \mathrm{O}_{19}$ & & 9,6 & 2,6 & & \\
\hline $\begin{array}{c}\mathrm{Gd}_{0.7} \mathrm{Yb}_{0.3} \\
\mathrm{MgAl}_{1} \mathrm{O}_{10}\end{array}$ & & 9,6 & 1,9 & & \\
\hline $\operatorname{LaLiAl}_{11} \mathrm{O}_{18.5}$ & & 10 & 3,8 & & \\
\hline \multicolumn{6}{|l|}{ Perovskites } \\
\hline \multicolumn{6}{|l|}{ Zirconatos } \\
\hline $\mathrm{BaZrO}_{3}$ & 2690 & 7,9 & 3,42 & 181 & \\
\hline $\mathrm{SrZrO}_{3}$ & 2800 & 10,9 & 2,3 & 170 & 1,5 \\
\hline \multicolumn{6}{|l|}{ Formas Complexas } \\
\hline $\mathrm{Ba}\left(\mathrm{Mg}_{1 / 3} \mathrm{Ta}_{2 / 3}\right) \mathrm{O}_{3}$ & 3100 & 10,9 & 2,71 & 186 & 0,7 \\
\hline$\underset{\mathrm{O}}{\mathrm{La}\left(\mathrm{Al}_{1 / 4} \mathrm{Mg}_{1 / 2} \mathrm{Ta}_{1 / 4}\right)}$ & & 9,7 & 1,82 & 174 & 0,6 \\
\hline $\mathrm{BaLa}_{2}^{3} \mathrm{Ti}_{3} \mathrm{O}_{10}$ & & $10-13$ & 0,7 & & \\
\hline
\end{tabular}


capacidade de deformação e por consequência tem-se uma maior resistência ao choque.

Apesar das perovskites apresentadas possuírem determinadas propriedades interessantes, não têm, contudo, uma tenacidade tão elevada quanto a da PSZ.

Nas perovskites complexas apresentadas nesta seç̧ão verifica-se que ambas possuem $\mathrm{Mg}$ na sua constituição, no entanto, é necessário ter em conta que este elemento volatiliza facilmente comparativamente com os outros o que pode levar, durante o processo APS, à deposição de um revestimento sem a estequiometria adequada e consequentemente a uma diminuição das propriedades destes materiais enquanto revestimentos. Estudos recentes têm vindo a procurar minimizar este efeito através da manipulação adequada dos parâmetros do processo APS, como por exemplo, o tempo de permanência das partículas no plasma [23].

$\mathrm{Na}$ perovskite $\mathrm{BaLa}_{2} \mathrm{Ti}_{3} \mathrm{O}_{10}$ não existe o problema da volatilização durante o processo de APS. Esta perovskite em camadas possui uma baixa condutividade térmica devido às suas ligações fracas entre os planos, apesar de as ligações dentro dos planos serem fortes. Isto leva a que a condução térmica seja maior dentro dos planos do que entre planos, em virtude do tipo de ligação existente. Para este material a capacidade como revestimento a temperaturas próximas de $1200^{\circ} \mathrm{C}$ é superior à da $\mathrm{PSZ}$, e pensa-se que este facto é devido à presença da já mencionada fragmentação da rede cristalina ocorrida no revestimento produzido via APS, sem que, no entanto, se tenha o já referido problema da volatilização levando à deposição de compostos não-estequiométricos [24]. Existe ainda a necessidade de se fazerem testes para compreender totalmente a capacidade deste material poder manter as propriedades necessárias para actuar como TBC a temperaturas superiores a $1200^{\circ} \mathrm{C}$.

\section{CONCLUSÕES}

Têm sido investigados diversos materiais com vista a substituir a PSZ como material dominante em aplicações para TBCs. Na Tabela I apresenta-se um resumo das propriedades termo-mecânicas de alguns dos materiais abordados neste trabalho e que são vistos como potenciais substitutos da PSZ em TBCs.

Entre os diferentes materiais estudados os pirocloros e os "cluster TBC" são aqueles que têm propriedades mais interessantes em virtude de possuírem baixos valores de condutividade térmica e elevados valores de coeficiente de expansão térmica simultaneamente. Além disso este tipo de materiais não apresenta problemas de maior relativamente ao seu processamento, o que é outro factor importante a ter em conta.

Espera-se o desenvolvimento futuro destes novos materiais com vista a verificar se existem realmente hipóteses para que a PSZ seja substituída por outros materiais em sistemas TBCs, possibilitando assim um aumento das temperaturas de trabalho, algo que é extremamente importante em turbinas, por exemplo, uma vez que leva a um aumento da eficiência.

\section{REFERÊNCIAS}

[1] X. Q. Cao, R. Vassen, D. Stoever, J. Eur. Ceram. Soc. 24 (2004) 1-10.

[2] R. Vassen, A. Stuke, D. Stover, J. Thermal Spray Tech. 18 (2008) 181-186.

[3] www.ul.ie/elements/Issue6/Gas\%20Turbine\%20Blades. htm, consultado em 18-04-2011.

[4]www.matsceng.ohio-state.edu/faculty/padture/ padturewebpage/padture/research.htm, consultado em 1804-2011.

[5] R. Miller, J. Thermal Spray Tech. 6 (1997) 35-42.

[6] J. R. Brandon, R. Taylor, Surface Coating Tech. 46 (1991) 75-90.

[7] A. Cipitria, I. O. Golosnoy, T. W. Clyne, Acta Mater. 57 (2009) 993-1003.

[8]www.doitpoms.ac.uk/tlplib/fuel-cells/printall.php, consultado em 18-04-2011.

[9] R. Vassen, F. Traeger, D. Stover, Int. J. Appl. Ceram. Tech. 1 (2004) 351-361.

[10] H. Chen, Y. Liu, Y. Gao, S. Tao, H. Luo, J. Am. Ceram. Soc. 93, 6 (2010) 1732-1740.

[11] R. Vassen, G. Pracht, D. Stover, Proc. Int. Thermal Spray Conf. (2002) 202-207.

[12] D. Zhu, R. Miller, D. Fox, Proc. 48 th AIAA/ASME/ ASCE/AHS/ASC Structures, Structural Dynamics, and Materials Conf. (2007) 1.

[13] som.web.cmu.edu/structures/S063-BaFe12O19.html, consultado em 18-04-2011.

[14] R. Collongues, D. Gourier, A. Kahn-Harari, A. Lejus, J. Thery, D. Vivien, Annual Review Mater. Res. 20 (1990) 51-82.

[15] G. Schäfer, R. Gadow, Int. Conf. Advanced Ceramics and Composites (1999) 291.

[16] C. Friedrich, R. Gadow, T. Schirmer, J. Thermal Spray Techn. 10 (2001) 592-598.

[17] L. Xie, A. Cormack, J. Solid State Chem. 88 (1990) 543-554.

[18] A. Doncieux, D. Stagnol, M. Huger, T. Chotard, C. Gault, T. Ota, S. Hashimoto, J. Mater. Sci. 43 (2008) 41674174.

[19]T. Negahdari, M. Gerdes, M. Willert-Porada, Mechanical Properties and Performance of Engineering Ceramics and Composites III, Willey-American Ceram. Soc. (2008) 15.

[20] C.-S. Hong, S.-Y. Chu, W.-C. Su, R.-C. Chang, H.-H. Nien, Y.-D. Juang, J. Appl. Phys. 101 (2007) 054117.

[21] R. Vassen, X. Cao, F. Tietz, D. Basu, D. Stover, J. Am. Ceram. Soc. 83 (2000) 2023-2028.

[22] W. Ma, M. Jarligo, D. Mack, D. Pitzer, J. Malzabender, R. Vassen, D. Stover, J. Thermal Spray Tech. 17 (2008) 831835.

[23] Y. Zhang, D. Mack M. Jarligo, X. Cao, R. Vassen, D. Stover, J. Thermal Spray Tech. 18 (2009) 694-701.

[24] H. Guo, H. Zhang, G. Ma, S. Gong, Surface Coating Tech. 204 (2009) 691-696.

(Rec. 18/04/2012, Ac. 08/06/2012) 